\title{
Combined influence of illness duration and medication type on visual sensitivity in schizophrenia
}

\author{
Natalia L. Almeida, (iD Thiago P. Fernandes, Eveline H. Lima, Hemerson F. Sales, Natanael A. \\ Santos
}

Laboratório de Percepção, Neurociências e Comportamento (LPNeC), Universidade Federal da Paraíba (UFPB), João Pessoa, PB, Brazil.

\begin{abstract}
Objective: Patients with schizophrenia have visual processing impairments. The main findings from the literature indicate that these deficits may be related to differences in paradigms, medications, and illness duration. This study is part of a large-scale study investigating visual sensitivity in schizophrenia. Here we aimed to investigate the combined effects of illness duration and antipsychotic use on contrast sensitivity function.

Methods: Data were collected from 50 healthy controls and 50 outpatients with schizophrenia (classified according to illness duration and medication type) aged 20-45 years old. The contrast sensitivity function was measured for spatial frequencies ranging from 0.2 to 20 cycles per degree using linear sine-wave gratings.

Results: Patients with an illness duration $>5$ years had more pronounced deficits. Differences in the combined effects of illness duration and antipsychotic use were marked in patients on typical antipsychotics who had been ill $>10$ years. No significant differences were found between typical and atypical antipsychotics in patients with an illness duration $<5$ years.

Conclusion: Visual impairment was related to both long illness duration and medication type. These results should be tested in further studies to investigate pharmacological mechanisms.
\end{abstract}

Keywords: Schizophrenia; contrast sensitivity function; illness duration; antipsychotics; psychiatry

\section{Introduction}

Schizophrenia (SCZ) is a debilitating disorder commonly related to impairments in several domains of cognition and perception. Visual processing, a doorway to cognition, can be non-invasively investigated the due to the anatomical position of the retina. Furthermore, the ways in which illness duration and medications influence vision have not been well established.

Mixed results in previous studies investigating the effects of medication on visual function in SCZ have led to more comprehensive research in recent years. Fernandes et al. ${ }^{1}$ investigated the effects of antipsychotics on a variety of visual and cognitive tasks. Patients on typical and atypical antipsychotics were carefully selected and tested in two studies. The results of both studies indicated that patients on typical antipsychotics performed worse than those on atypical antipsychotics for all visual tasks. Although there were no significant differences in cognitive measures, similar trends were found. However, the extent to which the mixed results are attributable to differences in medications and illness duration remain unknown. It should be pointed out that Shoshina \& Shelepin ${ }^{2}$ conducted an interesting precursor study on patients with

Correspondence: Natalia L. Almeida, Departamento de Psicologia, Centro de Ciências Humanas, Letras e Artes (CCHLA), Universidade Federal da Paraíba (UFPB), Campus I, Cidade Universitária, CEP 58051-90, João Pessoa, PB, Brazil.

E-mail: natalialalmeida@ hotmail.com

Submitted Nov 06 2018, accepted Mar 16 2019, Epub Jul 012019. different illness durations. They used spatial frequencies of $0.4,3.6$ and 17.9 cycles per degree (cpd) with Gabor patches, observing a reduced visual processing in patients who had been ill $>10$ years. Although patients on typical antipsychotics performed worse at contrast detection, those on atypical antipsychotics performed worse at medium spatial frequencies. However, these authors did not evaluate a wide range of spatial frequencies (which can provide a better description of how visual processing is impaired at low, medium and high spatial frequencies). Furthermore, there was no specific analysis on the combined effects of illness duration and medication type.

As an extension of the work of Fernandes et al., ${ }^{1}$ the present study sought to investigate the combined effects (interaction) of illness duration and medication type on visual impairment using a wide range of spatial frequencies. According to previous findings, contrast sensitivity function (CSF) has potential as a non-invasive diagnostic tool for clinical practice, being an alternative or adjuvant to existing tools and providing a quick and thorough investigation of initial visual processing. Understanding how antipsychotics and illness duration influence visual ability has both practical and clinical implications and may be useful as a diagnostic tool for prodromal phases.

How to cite this article: Almeida NL, Fernandes TP, Lima EH, Sales HF, Santos NA. Combined influence of illness duration and medication type on visual sensitivity in schizophrenia. Braz J Psychiatry. 2020;42:27-32. http://dx.doi.org/10.1590/1516-44462018-0331 
A total of 50 patients, treated with either a single typical $(n=25)$ or atypical $(n=25)$ antipsychotic medication and no other concurrent medication, were carefully selected for inclusion in this study.

\section{Materials and methods}

\section{Participants}

Fifty healthy controls (mean age $=32.5$ years, standard deviation $[\mathrm{SD}]=5.5$ years), and $50 \mathrm{SCZ}$ patients (mean age $=32.2$ years, $S D=5.9$ years), participated in this study. The patients were recruited from a communitybased outpatient clinic in João Pessoa, Brazil, having been diagnosed by psychiatrists at the same institution according to DSM-5 criteria. ${ }^{3}$ The exclusion criteria for SCZ patients were: a) antipsychotic polypharmacy; b) comorbidities; c) neurological disorders, such as intellectual disability; d) discontinuation of medication at any time; and e) tobacco use disorder. ${ }^{4}$ See below for additional exclusion criteria for all subjects. Further details about medication can be found in the precursor study. ${ }^{1}$ Chlorpromazine equivalents were calculated using previously described conversion factors. ${ }^{5-7}$

The healthy controls were recruited from the general population. They had no neuropsychiatric disorders according to the Structured Clinical Interview for the DSM-5. ${ }^{8}$ General exclusion criteria were: age $<25$ or $>48$ years old, current history of neurological disorder, cardiovascular disease, history of head trauma, history of contact with substances such as solvents, current or previous drug or substance abuse, and current use of medications that could affect visual processing and cognition (e.g., benzodiazepines).

The participants had no retinal abnormalities according to funduscopic examination or optical coherence tomography. All participants were screened for color blindness using the Ishihara test for color deficiency ${ }^{9}$ and had normal or corrected-to-normal vision (at least 20/20) as assessed by a Snellen chart. The Brief Psychiatric Rating Scale ${ }^{10}$ was used to evaluate illness severity.

\section{Stimuli and apparatus}

Contrast sensitivity was measured using Metropsis software, with an average luminance of $50 \mathrm{~cd} / \mathrm{m}^{2}$. All procedures were performed in a room kept at $26^{\circ} \mathrm{C} \pm 1^{\circ} \mathrm{C}$, whose walls were covered in gray to better control luminance during the experiments. The measurements were recorded using binocular vision at a distance of $150 \mathrm{~cm}$ from the computer monitor. The contrast sensitivity function was measured for spatial frequencies ranging from 0.2 to $20 \mathrm{cpd}$ using linear sine-wave gratings. The stimuli and apparatus have been described elsewhere (for details, see Fernandes et al. ${ }^{1,4,11}$ ).

\section{Procedure}

Prior to starting the tests, detailed task instructions were provided to the participants. Accuracy was emphasized over speed. A practice session was conducted to familiarize the participants with the procedure and avoid misunderstandings. Metropsis performs a validity check of the data with catch trials to detect random responses. The procedure was performed in a quiet, comfortable room; each visual measurement session lasted from 30 to 45 minutes. To avoid fatigue, the participants were encouraged to take breaks at their discretion after completing a reversal for any spatial frequency. The participants were instructed to focus on a small black cross in the center of the monitor. A two-alternative forced-choice method was used. The participants' task was to identify, using a remote-control response box, whether the grating was presented on the left or right side of the screen (Figure 1). A three-down/one-up staircase procedure with dynamic steps was used to derive a contrast threshold with a detection level of $79.4 \%$ for a psychometric function. ${ }^{12}$ The session ended after 12 contrast reversals occurred. Higher contrast sensitivity values indicated that the participant had higher sensitivity (for procedural details, see Fernandes et al. ${ }^{1,4,11,13}$ ).

\section{Statistical analysis}

Distributions for each group were compared using the Monte Carlo methods for skewness and kurtosis. ${ }^{14,15}$ The cutoff value was $>1.96$ for both kurtosis and skewness. Fractional rank (applying inverse cumulative distribution functions ${ }^{16}$ ) was used to transform outliers. In multivariate analysis of the CSF data, the groups were the betweensubject factor, while the spatial frequencies were the dependent variables. The assumption of variance homogeneity was not violated. To prevent inflation of type 1 errors, values of $p<0.006$ ( $p$-value/number of comparisons) were considered statistically significant. For categorical variables, the $\chi^{2}$ test was used. Bootstrapping is one of the most appropriate methods for a small sample size because it requires no assumption of the shape of the sampling distribution. Thus, all parametric tests were run using 5,000 bootstrapping resamples. ${ }^{17,18}$ Effect sizes $>0.50$ were considered medium-to-large.

In a separate analysis, a mean frequency of $0.2-2.0$ was classified as low spatial frequency (LSF), a mean of 3.1-6.0 was classified as a medium-to-high spatial frequency (MSF), and a mean of 8.8-20.0 was classified as a high spatial frequency (HSF).

Pearson's product-moment correlation ( $r$ ) and the pointbiserial correlation $(r)$ were used to assess the relationships between CSF data outcomes and biological and sociodemographic variables, such as age, gender, education level, illness duration, and illness severity. Moderation analysis and the bootstrapping method were applied. ${ }^{17}$ Model 1 of Hayes' PROCESS for SPSS with 5,000 bootstrap simulations was used. The bias-corrected and accelerated bootstrap confidence intervals (BCas) are also presented. Illness duration and antipsychotic medication type were dummy coded and entered in the model. The regression assumptions were checked first. The data presented normality, homoscedasticity, and no multicollinearity (tolerance values $<0.01$ ). The data are presented as mean and standard error of the mean. 


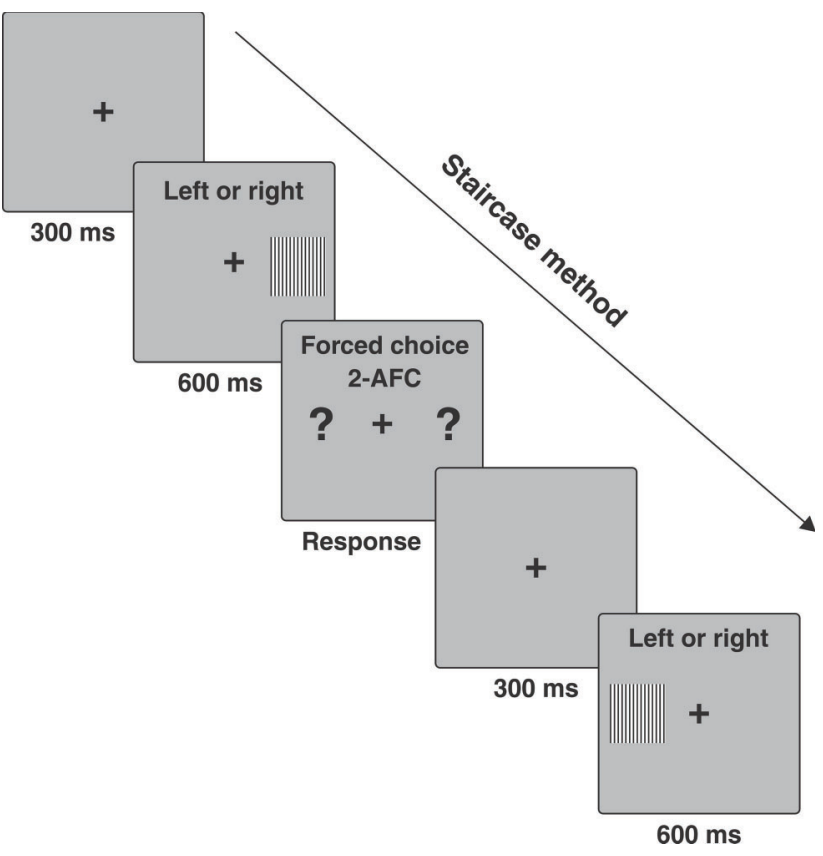

Figure 1 Contrast sensitivity function task. The participants' task was to identify, using a remote control response box, whether a grating stimulus appeared on the left or right side of a computer screen. Each stimulus had an exposure time of $600 \mathrm{~ms}$, with an intertrial interval of $300 \mathrm{~ms}$. Metropsis uses a psychophysical method to randomize spatial frequencies (low, medium, and high) and contrast values. $2-\mathrm{AFC}=$ twoalternative forced-choice.

\section{Ethics statement}

The present study followed the ethical principles of the Declaration of Helsinki and was approved by the Universidade Federal da Paraíba ethics committee (CCAE 45774715.9.0000.5188). Written informed consent was obtained from all participants.

\section{Results}

\section{Participants}

The sample's characteristics are summarized in Table 1. The groups did not differ in age, education level, or the ratio of males to females. No significant correlations were found between chlorpromazine equivalents and CSF measures in the SCZ groups.

\section{Contrast sensitivity function}

To obtain a baseline, both of the SCZ groups were compared with the control group. A statistically significant difference in CSF was found for all spatial frequencies (all $p<0.001$; Figure 2). The data described below were compared with control curves.

\section{Illness duration}

There was a statistically significant difference in illness duration between groups $\left(\mathrm{F}_{24,273}=7.332, p<0.001\right.$;
Table 1 Participant characteristics

\begin{tabular}{lcc}
\hline Variable & $\mathrm{HC}(\mathrm{n}=50)$ & $\mathrm{SCZ}(\mathrm{n}=50)$ \\
\hline Gender, $\mathrm{n}$ & & \\
$\quad$ Male & 31 & 36 \\
$\quad$ Female & 19 & 14 \\
& & \\
Age (years) & $32.5(5.5)$ & $32.2(5.9)$ \\
Education level (years) & $11.4(2.1)$ & $9.1(1.6)$ \\
Age of onset (years) & - & $20.0(2.5)$ \\
Number of hospitalizations & - & $6.5(3.1)$ \\
Comorbidities & - & - \\
& & \\
Illness duration, $\mathrm{n}$ & & 14 \\
$\quad<5$ years & & 19 \\
6-9 years & & 17 \\
$>10$ years & &
\end{tabular}

Brief Psychiatric Rating Scale score 41.3 (3.8) Chlorpromazine equivalent (mg) 685 (340)

Data presented as mean (standard deviation), unless otherwise specified.

$\mathrm{HC}=$ healthy controls; $\mathrm{SCZ}=$ patients with schizophrenia.

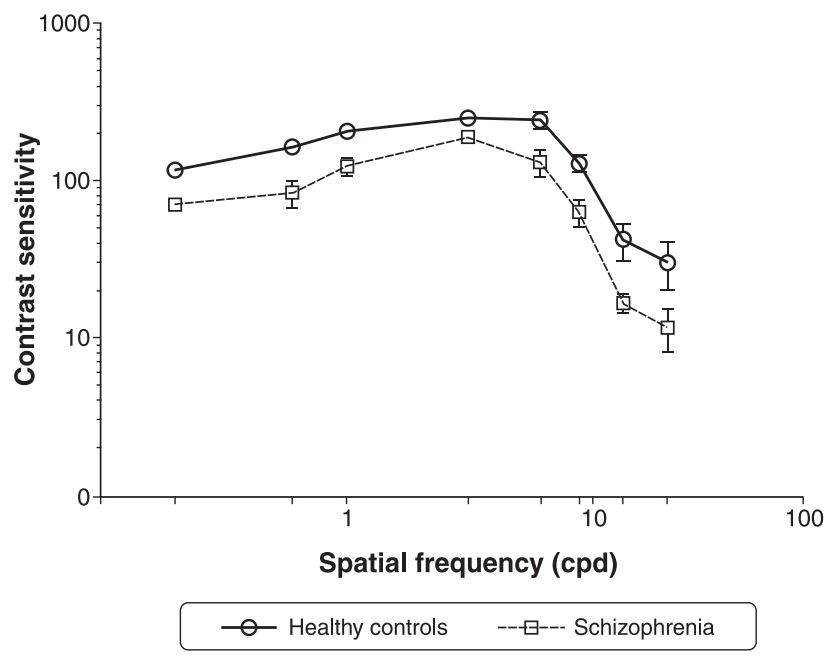

Figure 2 Contrast sensitivity curves as a function of spatial frequency (cycles per degree [cpd]) in healthy controls and patients with schizophrenia. Each data point represents sensitivity (reciprocal of contrast threshold). Error bars represent the standard error of the mean.

Pillai's trace $=1.176$, partial $\omega 2=0.338[95 \%$ confidence interval: 0.265 to 0.407$])$. Post-hoc tests revealed similarity between the CSF curves in the SCZ groups with $<5$ years and 5-9 years of illness duration for all spatial frequencies, with the exception of $0.2 \mathrm{cpd}(\mathrm{p}=0.035$; 95\% BCas: -17.511 to -10.601$), 0.5 \mathrm{cpd}(\mathrm{p}=0.037 ; 95 \%$ BCas: -18.189 to -5.455$), 1.0 \mathrm{cpd}(\mathrm{p}=0.039 ; 95 \%$ BCas: -15.466 to -4.564$), 13.2 \mathrm{cpd}(\mathrm{p}<0.001 ; 95 \%$ BCas: -30.755 to -17.730 ), and $20.0 \mathrm{cpd}(\mathrm{p}<0.001 ; 95 \%$ BCas: -27.722 to -14.460 ). The SCZ group with $>10$ years of illness duration had a lower CSF curve than the other groups for all spatial frequencies (all $p<0.001$; Figure 3 ). The mean data for LSF, MSF, and HSF were: SCZ group with $<5$ years of illness duration (124.87 \pm 17.34 , $174.55 \pm 7.34$, and $36.45 \pm 3.76$, respectively), SCZ group with 5-9 years of illness duration $(96.70 \pm 8.57$, 


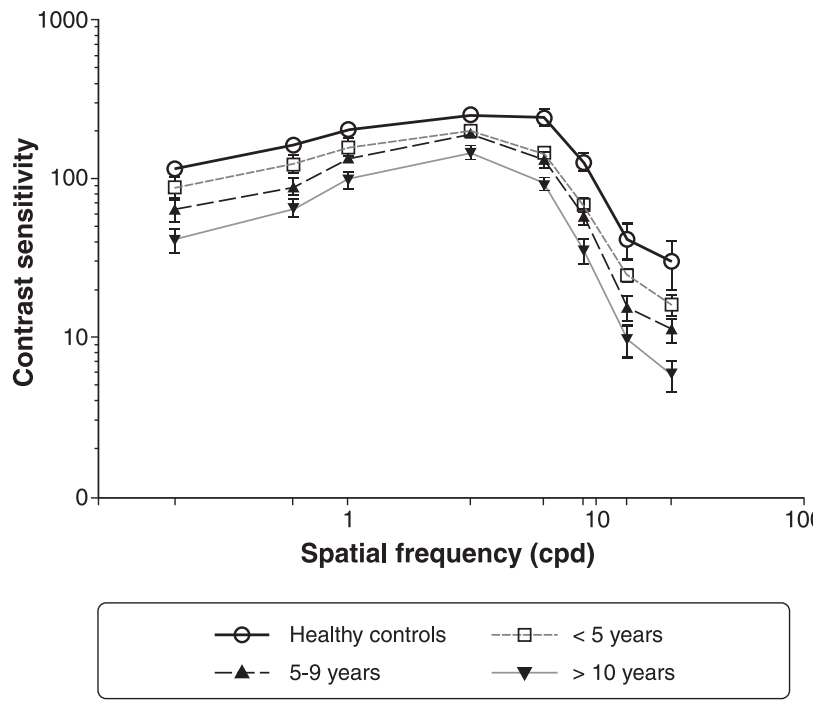

Figure 3 Contrast sensitivity curves as a function of spatial frequency (cycles per degree [cpd]) in healthy controls and patients with schizophrenia classified according to the illness duration. Each data point represents the sensitivity (reciprocal of contrast threshold). Error bars represent the standard error of the mean.

164.11 \pm 12.15 , and $29.26 \pm 4.15$, respectively), and SCZ group with $>10$ years of illness duration $(68.90 \pm 8.45$, $121.04 \pm 10.76$, and $17.20 \pm 3.30$, respectively).

Interaction between medication type and illness duration

The following data show CSF curves for the SCZ group, divided into clusters of illness duration and medication type (typical vs. atypical antipsychotics). A statistically significant difference was found between groups $\left(\mathrm{F}_{16,174}=4.347\right.$, $\mathrm{p}<0.001$; Pillai's trace $=0.571$, partial $\omega 2=0.219[95 \%$ confidence interval: 0.132 to 0.311$])$. Post-hoc tests revealed that, when controlling for medication, the group of patients who used typical antipsychotics had lower overall CSF curves for all spatial frequencies for 5-9 years (all $p<0.01$ ) and $>10$ years (all $p<0.001$ ) of illness duration. The CSF measurements are presented in Figure 4.

\section{Correlation analysis in schizophrenia groups}

Correlation analyses were conducted to investigate relationships between the biological and sociodemographic data and CSF results. Separate analyses were performed. No significant relationships were found between any of the pairs of variables in the control group (all $p>0.05)$. In the SCZ group, no significant relationships were found between age and performance at different spatial frequencies (all $p>0.05$ ) or between education level and performance at any spatial frequency (all $p>0.05$ ). Significant relationships were found between illness duration and LSF $(r=0.831, p<0.001 ; 95 \%$ BCas: 0.785 to 0.870$)$ and illness duration and HSF $(r=0.606$, $p<0.001 ; 95 \%$ BCas: 0.480 to 0.726 ). No significant relationship was found between illness duration and MSF in the SCZ group $(r=0.145, p=0.151 ; 95 \%$ BCas:
-0.029 to 0.316 ). Partial correlations were performed by controlling for medication type, indicating a possible relationship between longer illness duration and lower visual performance. A large negative correlation was found for LSF $(r=-0.637, p<0.001 ; 95 \%$ BCas: -0.752 to 0.491 ), and a small negative correlation was found for HSF ( $r=-0.212, p=0.036$; $95 \%$ BCas: 0.004 to $0.106)$.

\section{Moderation analysis}

A moderation analysis was conducted to test whether antipsychotic type moderated the relationship between illness duration and the LSF detection outcome (Figure 5).

A mitigating effect was apparent in the interaction data: with increased illness duration and according to antipsychotic type, LSF detection decreased. LSF detection was lower with typical antipsychotics than atypical antipsychotics. The interaction was significant in the model $\left(F_{3,146}=59.20, p<0.001\right.$, adjusted $\left.R^{2}=0.64\right)$. All of the coefficients were significant $\left(p<0.01, R^{2}\right.$ change $=$ $0.025, p=0.010)$. The following equation represents the model: LSF $=30.44-14.14$ (illness duration) - 21.99 (antipsychotic type) +9.55 (interaction) (Table 2).

\section{Discussion}

The present study investigated whether the combined effects of illness duration and antipsychotic type influence visual sensitivity in SCZ patients. The results supported our main hypothesis that SCZ may be associated with impairments in visual processing. More specifically, patients whose illness duration was $<10$ years and $<5$ years performed better than those whose illness duration was $>10$ years. These differences were pronounced in patients who used typical antipsychotics. One novel aspect of the present study was the investigation of illness duration in patients who took the same type of medication for the entire duration of the illness. Another aspect was our use of moderation analysis, in which antipsychotic type was found to interact with illness duration. Overall, the SCZ patients had lower sensitivity to all spatial frequencies, but illness duration was related to a decrease in both LSF and HSF (Figures 2 and 3). There was an interaction between these variables only for LSFs, which requires further investigation.

The establishment of correlations between SCZ and visual processing does not exclude the importance of understanding the interactive role of antipsychotic type and illness duration. Differences in the responsivity of visual pathways due to chronic antipsychotic use and illness duration can bias visual processing toward the cortex, thus impairing vision. Although this may be attributable to a hemispheric difference in SCZ patients that involves both the ventral and dorsal streams, ${ }^{19}$ this possibility remains unclear. Our results indicated both LSF and HSF impairment, suggesting that medication alone cannot account for the abnormal findings in SCZ patients, and illness duration may play an important role (e.g., cumulative effects of medication may impair visual pathways). Hence, this interaction may reduce visual processing. It could be argued that HSF 
A
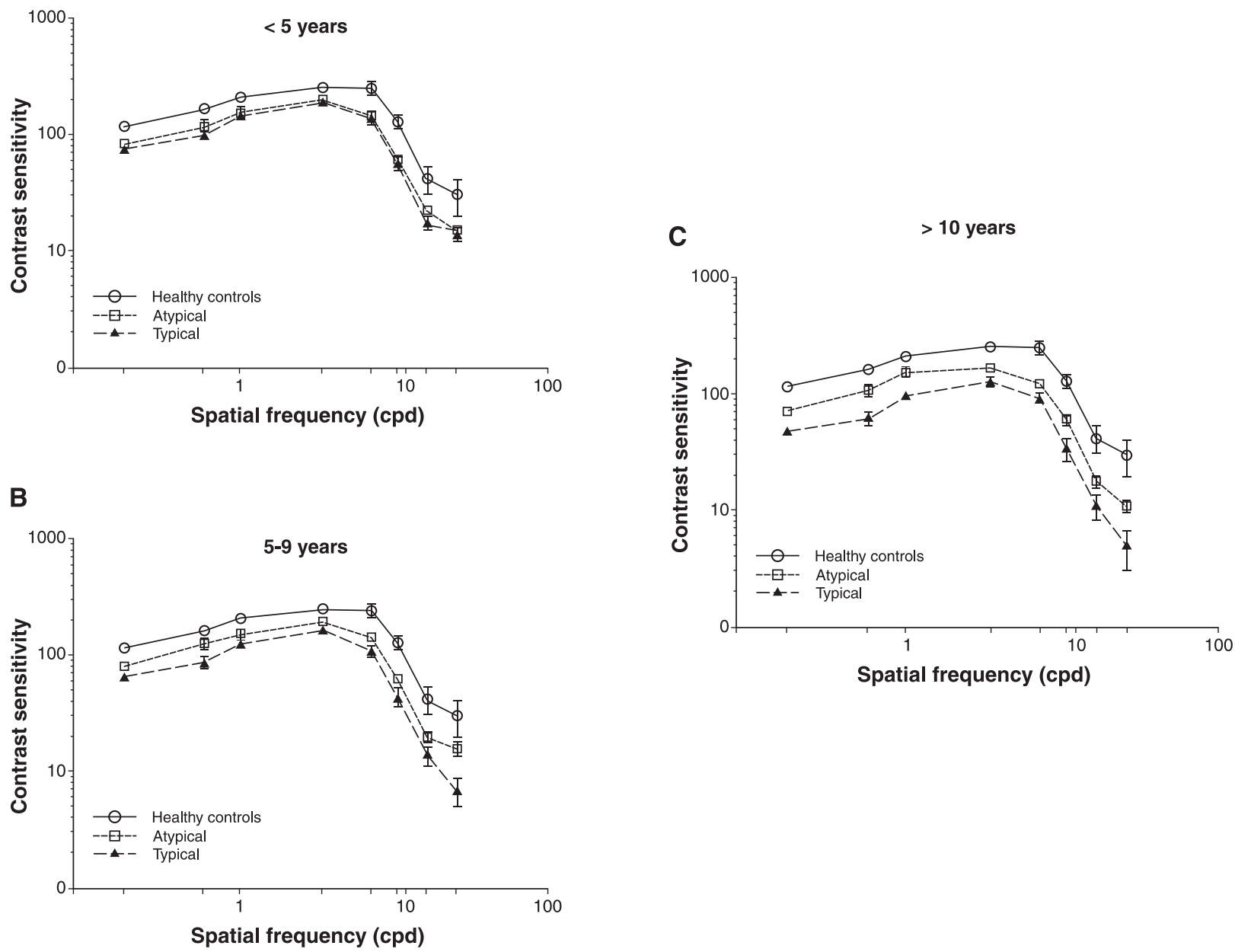

Figure 4 Contrast sensitivity curves as a function of spatial frequency (cycles per degree [cpd]) in healthy controls and patients with schizophrenia classified according to medication type, with an illness duration of (A) $<5$ years, (B) 5-9 years, and (C) $>10$ years. Each data point represents sensitivity (reciprocal of contrast threshold). Error bars represent the standard error of the mean.

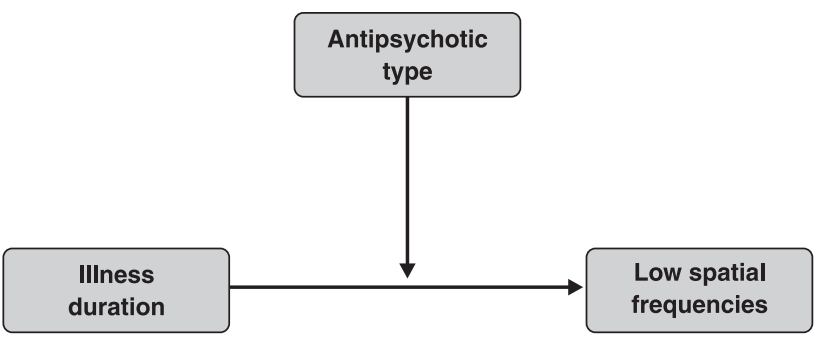

Figure 5 Interaction diagram of the relationship between the moderating variable, illness duration, and outcome for low spatial frequencies. impairment may reflect lower visual acuity, in addition to or even in the absence of true deficits in contrast sensitivity. However, we observed no differences in visual acuity, which was screened using Snellen's chart with a cutoff of 20/20. This also should be investigated in further studies, although previous findings have revealed the same pattern.

It is important to point out that establishing correlations between SCZ, medication type, illness duration, and visual processing is difficult. For example, the use of different medications has been a confounding factor in several previous studies. $^{4,20,21}$ The exact mechanism of the medications'

Table 2 Linear models of predictors of visual performance

\begin{tabular}{lccrr}
\hline & $\mathrm{b}$ & $\mathrm{SE} \mathrm{B}$ & $t$ & $\mathrm{p}$-value \\
\hline Constant & 30.44 & 9.31 & 2.64 & 0.001 \\
Illness duration & -14.14 & 3.55 & 3.49 & 0.001 \\
Antipsychotic type & -21.99 & 1.33 & 2.87 & 0.009 \\
Illness duration vs. antipsychotic type & 9.55 & .11 & 4.42 & 0.001 \\
\hline
\end{tabular}

$\mathrm{R}^{2}=0.64$. 
adverse effects and its relationship with illness duration are not fully understood. As mentioned above, some studies on this issue have used different methods and paradigms but have yielded similar results. In view of this, we suggest that different classes of the same antipsychotic type can have more influence than illness duration per se. Most of the atypical antipsychotics used by our patients were from the benzisoxazole group, which does not vary substantially in mechanism of action, suggesting that this was not an intervening variable. Similarly, most of the typical antipsychotics were from the same class (i.e., phenothiazines). However, without a group of unmedicated patients, the results should be carefully interpreted, as reported by Fernandes et al. ${ }^{1}$ It is important to reiterate that dividing patients into typical and atypical groups is usually an oversimplification, since most atypical antipsychotics have different receptor profiles (for example NMDA receptors ${ }^{22}$ ).

In this study we investigated whether medication type influences visual sensitivity after years of treatment. Our results indicated that, independent of medication type, visual processing was impaired after 5 years of using the same drug. This impairment was more pronounced after 10 years of using the same drug (Figures 4 and 5), which was demonstrated by our moderation analysis. Although we were unable to independently confirm that all patients used the same medication for all these years, the patients were followed up for approximately 4 years and their medical records were evaluated. Nevertheless, we sought to minimize the intervening variables regarding medication effects and illness duration (such as differences in body mass index, for example). This is an important issue that should be examined further in future studies.

Although this may be an over-extrapolation of the data, the detection of subtle changes in visual processing in the early stages of the disease could guide antipsychotic therapy. Perceptual decrements can be observed before the onset of the pathology and, hence, antipsychotic intake, and this can be assessed using optical coherence tomography as a biomarker, for example. ${ }^{23}$ Such investigations would allow comparisons between controlled and nonrandomized trials. Considering that not all SCZ patients respond equally to antipsychotics or are equally vulnerable to side effects, noninvasive methods of early visual processing assessment in clinical practice could be a promising way to improve patient prognosis.

In summary, the present results showed that differences in visual sensitivity are pronounced in SCZ patients on typical antipsychotics whose illness duration is greater than 5 years.

\section{Acknowledgements}

This study received financial support from Coordenação de Aperfeiçoamento de Pessoal de Nível Superior (CAPES; grant 001) and from Conselho Nacional de Desenvolvimento Científico e Tecnológico (CNPq; grant 309778/2014-0).

\section{Disclosure}

The authors report no conflicts of interest.

\section{References}

1 Fernandes TP, Shaqiri A, Brand A, Nogueira RL, Herzog MH, Roinishvili M, et al. Schizophrenia patients using atypical medication perform better in visual tasks than patients using typical medication. Psychiatry Res. 2019;275:31-8.

2 Shoshina II, Shelepin YE. Contrast sensitivity in patients with schizophrenia of different durations of illness. Neurosci Behav Physiol. 2015;45:512-6.

3 American Psychiatric Association. Diagnostic and Statistical Manual of Mental Disorders, Fifth Edition (DSM-5). Arlington: American Psychiatric Publishing; 2013.

4 Fernandes TM, de Andrade MJ, Santana JB, Nogueira RM, Dos Santos NA. Tobacco use decreases visual sensitivity in schizophrenia. Front Psychol. 2018;9:288.

5 Danivas V, Venkatasubramanian G. Current perspectives on chlorpromazine equivalents: comparing apples and oranges! Indian J Psychiatry. 2013;55:207-8.

6 Kreyenbuhl J, Buchanan RW, Dickerson FB, Dixon LB; Schizophrenia Patient Outcomes Research Team (PORT). The Schizophrenia Patient Outcomes Research Team (PORT): updated treatment recommendations 2009. Schizophr Bull. 2010;36:94-103.

7 Woods SW. Chlorpromazine equivalent doses for the newer atypical antipsychotics. J Clin Psychiatry. 2003;64:663-7.

8 American Psychiatric Association. Structured clinical interview for DSM-5 (SCID-5) [Internet]. 2015 [cited 2019 Mar 28]. www.appi.org/ products/structured-clinical-interview-for-dsm-5-scid-5

9 Ishihara S. The series of plates designed as a test for colourblindness. Tokyo: Kanehara Shuppan; 1972.

10 Overall JE, Gorham DR. The brief psychiatric rating scale. Psychol Rep. 1962;10:799-812.

11 Fernandes TM, Silverstein SM, Almeida NL, Santos NA. Psychophysical evaluation of contrast sensitivity using Gabor patches in tobacco addiction. J Clin Neurosci. 2018;57:68-73.

12 Levitt H. Transformed up-down methods in psychoacoustics. J Acoust Soc Am. 1971;49:Suppl 2: 467+.

13 Fernandes TM, Almeida NL, Santos NA. Effects of smoking and smoking abstinence on spatial vision in chronic heavy smokers. Sci Rep. 2017;7:1690.

14 Antonius R. Interpreting quantitative data with SPSS. Thousand Oaks: SAGE; 2003.

15 Tabachnick BG, Fidell LS. Using multivariate statistics. 5th ed. Boston: Allyn \& Bacon/Pearson Education; 2007.

16 Beasley TM, Erickson S, Allison DB. Rank-based inverse normal transformations are increasingly used, but are they merited? Behav Genet. 2009;39:580-95.

17 Hayes AF. Introduction to mediation, moderation, and conditional process analysis: a regression-based approach. New York: Guilford Press; 2013.

18 Härdle W, Bowman AW. Bootstrapping in nonparametric regression: local adaptive smoothing and confidence bands. J Am Stat Assoc. 1988;83:102-10.

19 Plomp G, Roinishvili M, Chkonia E, Kapanadze G, Kereselidze M, Brand A, et al. Electrophysiological evidence for ventral stream deficits in schizophrenia patients. Schizophr Bull. 2013;39:547-54.

20 Chen Y, Levy DL, Sheremata S, Nakayama K, Matthysse S, Holzman PS. Effects of typical, atypical, and no antipsychotic drugs on visual contrast detection in schizophrenia. Am J Psychiatry. 2003;160: 1795-801.

21 Fernandes TM, Andrade SM, de Andrade MJ, Nogueira RM, Santos NA. Colour discrimination thresholds in type 1 bipolar disorder: a pilot study. Sci Rep. 2017;7:16405.

22 Li M, Spaulding WD. The neuropsychopathology of schizophrenia: molecules, brain systems, motivation, and cognition. New York: Springer; 2016.

23 Silverstein SM, Paterno D, Cherneski L, Green S. Optical coherence tomography indices of structural retinal pathology in schizophrenia. Psychol Med. 2018;48:2023-33. 